\title{
Versión española del Cuestionario Disejecutivo (DEX-Sp): propiedades psicométricas en adictos y población no clínica
}

\section{Spanish version of the Dysexecutive Questionnaire (DEX-Sp): psychometric properties in addicts and non-clinical sample}

\author{
Eduardo José Pedrero Pérez*; José María Ruiz \\ SÁnCHEZ DE LEÓN ${ }^{\star \star} ;$ GLORIA ROJO MOTA ${ }^{\star \star *}$; MARCOS \\ LLANERO LUQUE ${ }^{\star \star \star *}$; ÁlVARO OLIVAR ArROYO ${ }^{\star \star \star \star \star}$; \\ José Carlos Bouso SalZ ${ }^{\star \star \star \star \star \star}$; Carmen Puerta \\ GARCíA $^{\star \star \star \star \star \star \star ~}$
}

\begin{abstract}
* Doctor en Psicología. CAD 4 San Blas. Instituto de Adicciones. Madrid Salud. Ayuntamiento de Madrid. ** Doctor en Psicología. Centro de Deterioro Cognitivo. Instituto de Salud Pública. Madrid Salud. Ayuntamiento de Madrid. ${ }^{\star \star \star}$ Terapeuta Ocupacional. CAD 4 San Blas. Instituto de Adicciones. Madrid Salud. Ayuntamiento de Madrid. Universidad Rey Juan Carlos. ${ }^{\star \star \star \star}$ Neurólogo. Centro de Deterioro Cognitivo. Instituto de Salud Pública. Madrid Salud. Ayuntamiento de Madrid. ${ }^{\star \star \star \star \star}$ Diplomado en Educación Social. Consejería de Educación. Comunidad de Madrid. ${ }^{\star \star \star \star \star \star ~ P s i c o ́ l o g o . ~ C e n t r o ~}$ de Investigación de Medicamentos. Hospital de la Santa Creu y Sant Pau, Barcelona. ${ }^{\star \star \star \star \star \star \star}$ Médico. Directora del CAD 4 San Blas. Instituto de Adicciones. Madrid Salud. Ayuntamiento de Madrid.

Enviar correspondencia a:

Enviar correspondencia a: Eduardo J. Pedrero Pérez. C/ Alcalá, 527. 28027 Madrid, España. Tel.: 609587233. Fax: 917434775.

Correo e.: ejpedrero@yahoo.es
\end{abstract}

\section{RESUMEN}

Objetivo: Diversos estudios han evidenciado el deterioro de las funciones ejecutivas asociado al abuso de sustancias. Este hecho sugiere la conveniencia de contar con algún instrumento de cribado que sirva para establecer una línea base sobre los déficits que presentan los sujetos que inician tratamiento. El Cuestionario Disejecutivo (DEX) es un test ampliamente utilizado para estimar disfunción ejecutiva. El principal objetivo del presente trabajo se centra en estudiar las propiedades de la versión española del Cuestionario Disejecutivo (DEX-Sp). Complementariamente se pretende estudiar su potencial utilidad como instrumento de cribado en adictos en tratamiento.

Método: 131 sujetos de población no clínica y 127 adictos (con criterios DSM-IVTR para abuso o dependencia).

Resultados: Se estudia la consistencia interna $(\alpha$ de Cronbach $=0,91)$ entre otros indicadores de fiabilidad. El análisis factorial exploratorio y confirmatorio ofrece soluciones de 4 y 5 factores. Se estudió la dimensionalidad y el modelo estructural de base, asi como la validez convergente y discriminante. Se estudiaron las diferencias apreciadas entre adictos y población no clínica.

Conclusiones: Los datos sugieren que la versión española del DEX es un instrumento útil para evaluar sintomas disejecutivos generales, con la suficiente fiabilidad y validez para explorar deterioro cognitivo asociado al abuso de sustancias.

Palabras clave: Funciones ejecutivas, sindrome disejecutivo, lóbulo frontal, adicción a sustancias, cuestionario, análisis factorial, tratamiento.

\section{ABSTRACT}

Objective: Several studies have shown evidence of executive function impairment associated with substance abuse. This suggests the potential usefulness of a baseline assessment measure to screen for dysexecutive impairments in individuals beginning treatment. The Dysexecutive Questionnaire (DEX) is a test widely used to estimate executive dysfunction. The main objective of this work is to study the psychometric properties of the Spanish version of the Dysexecutive Questionnaire (DEX-Sp). A complementary goal is to study its potential utility for initial screening in addicted individuals seeking treatment.

Method: A sample of 131 non-clinical and 127 clinical individuals meeting the DSM-IV-TR substance abuse or dependence criteria were recruited.

Results: Internal consistency of the questionnaire was assessed (Cronbach's $\alpha$ $=0.91$ ), as well as other reliability indicators. Exploratory and confirmatory factor analysis offers four- and five-factor solutions. Also tested were the dimensionality and structural model and its convergent and discriminant validity with other instruments. Differences between clinical and non-clinical individuals and total scores were also analyzed.

Conclusions: The data suggest that the Spanish version of the DEX is a useful measure for assessing general symptoms of dysexecutive syndrome, and a valid, reliable and adequate screening test for estimating cognitive impairment associated with substance abuse.

Key words: Executive functions, dysexecutive syndrome, frontal lobe, addictive behaviour, questionnaires, psychometrics, factor analysis, treatment. 


\section{INTRODUCCIÓN}

Han sido descritos cinco circuitos paralelos, funcionalmente segregados en los lóbulos frontales (Alexander, DeLong y Strick, 1986) que Cummings (1993) y Mega y Cummings (1994) han vinculado a los sindromes comportamentales de origen frontal y clínicamente observables: el área motora suplementaria y los campos visuales frontales se asocian con disfunción motora (p.e., relacionados con reflejos primitivos, incontinencia), el córtex prefrontal dorsal se asocia con una típica disfunción cognitiva (p.e., pérdida de la capacidad de generar hipótesis y comprobarlas, inflexibilidad, desorganización, deterioro de la memoria de trabajo), el córtex prefrontal orbital lateral se asocia con dificultades en la autorregulación (p.e., desinhibición, impulsividad, pobreza en el juicio social) y el córtex frontal medial (el circuito cingulado anterior) se ha asociado con trastornos de la activación, el drive y la motivación.

El sindrome disejecutivo (habitualmente denominado sindrome del lóbulo frontal) se refiere a "un amorfo y variado grupo de déficits que son resultado de diversas etiologías, con diferentes localizaciones y una variable gama de alteraciones" (Stuss y Benson, 1984, p. 3). Estos déficits son normalmente evaluados con tests bien conocidos como el Stroop Test (Stroop, 1935), el Reitan Trail Making Test (Reitan, 1958) o el Wisconsin Card Sorting Test (Berg, 1948), aunque se dispone de algunos informes sobre pacientes con déficit clínicamente evidente en las funciones ejecutivas que ejecutan normalmente estas tareas (Eslinger y Damasio, 1985; Shallice y Burgess, 1991). Por otra parte, se han desarrollado pocos instrumentos psicométricos de medida de los cambios comportamentales, derivados de la disfunción ejecutiva, que son considerablemente desadaptativos (Malloy y Grace, 2005). Entre ellos se encuentran el Dysexecutive Questionnaire (DEX), el Frontal Systems Behavior Scale (FrSBe, Grace y Malloy, 2001), el Behavior Rating Inventory of Executive Functions (BRIEF, Gioia, Isquith, Guy y Kenworthy, 2000), y las lowa Rating Scales of Personality Change (IRSPC, Barrash, Tranel y Anderson, 2000). Se dispone también de algunas medidas desarrolladas para evaluar cambios comportamentales en demencia que pueden ser de utilidad en otros trastornos del sistema frontal, como el Frontal Behavioral Inventory (FBI, Kertesz, Davidson y Fox, 1997) y el Neuropsychiatric Inventory (NPI, Cummings, Mega, Gray, Rosenberg-Thompson, Carusi y Gornbein, 1994).

Diversos estudios han mostrado evidencia del deterioro del funcionamiento ejecutivo asociado al abuso de sustancias, como la heroína (Fishbein, Krupitsky, Flannery, Langevin, Bobashev, Verbitskaya et al., 2007; Prosser, Cohen, Steinfeld, Eisenberg, London y Galynker, 2006), cocaina (Forcada, Pardo y Bondía, 2006; Hester y Garavan, 2004), éxtasis (Schilt, de Win, Koeter, Jager, Korf, van den Brink et al., 2007), alcohol (Landa, Fernández-Montalvo y Tirapu, 2004; Landa, Fernández-Montalvo, Tirapu, LópezGoñi, Castillo y Lorea, 2006) o policonsumos (Giancola y Mezzich, 2003; Verdejo y Pérez-García, 2007). Los resultados obtenidos en estos estudios muestran que puede ser útil contar con un instrumento de evaluación de línea base que permita desarrollar un screening inicial de déficit en las funciones ejecutivas en sujetos que acceden a un tratamiento por problemas derivados del uso de drogas. La existencia de alteraciones neuropsicológicas puede comprometer la capacidad de estos individuos para asimilar los contenidos de programas con un alto componente educativo o cognitivo, y para participar e integrarse en las actividades terapéuticas de estos programas (Aharonovich et al., 2006; Aharonovich et al., 2003; Teichner et al., 2002; Verdejo, López-Torrecillas, Orozco y Pérez-García, 2002). Este cribado inicial podría preceder y facilitar una evaluación más profunda y específica de los déficits en las funciones ejecutivas, así como permitir la secuenciación de intervenciones específicas y actividades a lo largo del tratamiento (p.e., psicoterapia, terapia ocupacional, educación para la salud, intervenciones centradas en la familia).

El Cuestionario Disejecutivo (DEX) forma parte de la bateria Behavioural Assessment of the Dysexecutive Syndrome (BADS, Wilson, Alderman, Burgess, Emslie y Evans, 1996). La BADS consta de 6 tests diseñados para evaluar los efectos del síndrome disejecutivo que procuran eludir la pobre validez ecológica habitual en las medidas neuropsicológicas clásicas. A partir de estos 6 tests primarios se calcula un perfil: Rule Shift Cards (tendencias perseverativas o flexibilidad mental), Action Programme (capacidad para concebir y poner en práctica una solución a un problema práctico), Key Search (capacidad para planificar una estrategia para resolver un problema), Temporal Judgement (juicio y pensamiento abstracto basado en conocimientos), Zoo Map (capacidad para formular y desarrollar un plan) y Modified Six Elements (capacidad para manejar el tiempo). EI DEX es un cuestionario de 20 ítems suplementario a la batería BADS que no se utiliza para calcular el perfil. Tiene dos versiones: una que es cumplimentada por el propio sujeto (el DEX) y otra que cumplimenta alguna persona de su entorno próximo y significativo, preferiblemente con un contacto diario (DEX-R). Las puntuaciones en el DEX-R se consideran más válidas que las obtenidas por el propio sujeto, teniendo en consideración la pobre conciencia de problema típica en este tipo de pacientes (Burgess, Alderman, Evans, Emslie y Wilson, 1998). No obstante, algunos trabajos han encontrado que el autoinforme mediante el DEX es de utilidad para discriminar entre bebedores excesivos de alcohol y bebedores de dosis bajas y controles no bebedores (Heffernan, Ling y Bartholomew, 2004) y que es un buen predictor de problemas relacionados con el consumo de alcohol (Whitney, Hinson y Jameson, 2006) y otras sustancias (Reay, Hamilton, Kennedy y Scholey, 2006). Además, el DEX ha mostrado capacidad para predecir respuestas impulsivas (Hinson, Jameson y Whitney, 2003) estrechamente relacionadas con la adicción a sustancias (Verdejo, Lawrence y Clark, 2008). En consecuencia, la versión autoadministrada del DEX parece ofrecer un significativo valor cuando menos como screening preliminar de sintomatología disejecutiva.

El cuestionario DEX ha sido aplicado en el estudio de diferentes poblaciones, incluyendo a sujetos con el Síndrome de Prader-Willi (Walley y Donaldson, 2005), Enfermedad de 
Parkinson (Mathias, 2003), Alzheimer (Cullen, Coen, Lynch, Cunningham, Coakley, Robertson et al., 2005), lesión cerebral (Alderman, Dawson, Rutterford y Reynolds, 2001; Hart, Whyte, Kim y Vaccaro, 2005; Larson, Perlstein, Demery y Stigge-Kaufman, 2006; Wood y Liossi, 2006), y otros, como epilepsia, cáncer y esclerosis múltiple (Chaytor, SchmitterEdgecombe y Burr, 2006), así como en población no clínica (Amieva, Phillips y Della Sala, 2003; Chan, 2001; Chan, Hoosain y Lee, 2002; Wang, Chan y Deng, 2006; Mooney, Walmsley y McFarland, 2006).

El principal objetivo del presente estudio es explorar las propiedades psicométricas de la versión española del Cuestionario Disejecutivo (DEX) en dos submuestras, una de población no clínica y otra compuesta por sujetos adictos que comienzan tratamiento por su adicción. Como objetivo secundario se persigue el estudio de su potencial aplicabilidad como instrumento de screening inicial en los programas de tratamiento de las adicciones.

\section{MÉTODO}

\section{Participantes y procedimiento}

Se utilizó el método de Snowball Sampling (Goodman, 1961) para reclutar la submuestra de población no clínica. La submuestra clínica se compuso a partir de sujetos que demandaron tratamiento en un centro ambulatorio específico, de carácter público y gratuito (CAD 4, Instituto de Adicciones. Ayuntamiento de Madrid), por problemas derivados del uso de sustancias. Para su inclusión en el presente estudio, cada sujeto debía cumplir los criterios DSM-IV-TR para abuso o dependencia de al menos una sustancia en la evaluación rutinaria. Los participantes respondieron el cuestionario tras al menos dos semanas desde su incorporación al tratamiento para reducir la probabilidad de que se presentaran sintomas o déficits asociados con los efectos agudos de la intoxicación o la abstinencia. Se efectuaron controles toxicológicos de orina que permitieran controlar y descartar el uso de drogas durante todo el periodo de abstinencia. Los datos incluidos en este trabajo se obtuvieron con la conformidad con los usuarios, de acuerdo a la regulación del Instituto de Adicciones y de acuerdo a los principios de la Declaración de Helsinki. Finalmente, 131 sujetos de población no clínica y 127 de población clínica fueron reclutados; con anterioridad a su inclusión en el estudio, todos los sujetos firmaron un Consentimiento Informado. Los descriptivos de ambas submuestras se presentan en la Tabla 1.

\section{Instrumentos}

El Cuestionario Disejecutivo (DEX; Wilson et al., 1996) es un cuestionario de 20 ítems que se utiliza como suplemento de los tests primarios de la BADS. Los 20 items evalúan: problemas en el pensamiento abstracto, impulsividad, fabulación, problemas de planificación, euforia, problemas de secuenciación temporal, falta de insight, apatía, desinhibición, dificultades en el control de los impulsos, respuestas afectivas superficiales, agresión, falta de interés, perseveración, inquietud, falta de habilidad para inhibir respuestas, disociación entre conocimiento y respuesta, distractibilidad, pobre habilidad en la toma de decisiones y falta de interés por las reglas sociales. Cada item se puntúa en una escala tipo Likert de 5 puntos, entre "nunca" y "con mucha frecuencia". EI análisis factorial original reveló la existencia de 5 factores ortogonales: inhibición, intencionalidad, memoria ejecutiva, y dos factores relacionados con la emocionalidad y los cambios de personalidad denominados afecto positivo y negativo (Burgess et al., 1998). Los primeros 3 factores se relacionaron con puntuaciones en test neuropsicológicos. Los factores 4 y 5 no mostraron semejante relación; sin embargo, algunos aspectos importantes del funcionamiento neurocognitivo no son evaluados por los test de ejecución neuropsicológica. La traducción del DEX al idioma español se llevo a cabo siguiendo el procedimiento de traducciónretraducción paralela (Brislin, 1986). En el presente estudio solo se utilizó la forma autoinformada del DEX.

Tabla 1. Descriptivos de la muestra

\begin{tabular}{|c|c|c|c|c|c|c|c|}
\hline \multirow[b]{2}{*}{ No clínica } & \multirow[t]{2}{*}{$\mathrm{N}$} & \multicolumn{2}{|c|}{ Edad } & \multicolumn{4}{|c|}{ Nivel académico } \\
\hline & & Media & D.t. & Primaria & Secundaria & Avanzada & Universitaria \\
\hline Varones & $30(23 \%)$ & 30 & 11,7 & $2(6,7 \%)$ & $3(10,0 \%)$ & $4(13,3 \%)$ & $21(70,0 \%)$ \\
\hline Mujeres & $101(77 \%)$ & 101 & 9,2 & $6(5,9 \%)$ & $7(6,9 \%)$ & $28(27,7 \%)$ & $60(59,4 \%)$ \\
\hline \multicolumn{8}{|l|}{ Clínica } \\
\hline Varones & $104(82 \%)$ & 104 & 9,1 & $22(21,2 \%)$ & $43(41,3 \%)$ & $22(21,2 \%)$ & $17(16,3 \%)$ \\
\hline Mujeres & $23(18 \%)$ & 23 & 10,6 & $3(13,0 \%)$ & $5(21,7 \%)$ & $7(30,4 \%)$ & $8(34,8 \%)$ \\
\hline \multicolumn{8}{|l|}{ Droga principal } \\
\hline Heroina & 14 & 37,1 & 7,1 & $1(7,1 \%)$ & $9(64,3 \%)$ & $3(21,4 \%)$ & $1(7,1 \%)$ \\
\hline Cocaina & 48 & 34,5 & 8,3 & $8(16,7 \%)$ & $18(37,5 \%)$ & $12(25,0 \%)$ & $10(20,8 \%)$ \\
\hline Alcohol & 57 & 42,5 & 9,1 & $15(26,3 \%)$ & $20(35,1 \%)$ & $13(22,8 \%)$ & $9(15,8 \%)$ \\
\hline Cannabis & 5 & 31,2 & 10,7 & $0,0 \%$ & $1(20,0 \%)$ & $1(20,0 \%)$ & $3(60,0 \%)$ \\
\hline Éxtasis & 1 & 47 & , & --- & --- & --- & $1(100 \%)$ \\
\hline Benzodiacepinas & 2 & 40 & 7,1 & $1(50,0 \%)$ & --- & --- & $1(50,0 \%)$ \\
\hline
\end{tabular}


Escala de Comportamiento del Sistema Frontal (FrSBe; Grace et al, 1999; Grace y Malloy, 2001): es una escala de 46 items, dividida en 3 subescalas derivadas del análisis factorial: Apatía (FRSBEa), Desinhibición (FRSBEd) y Disfunción Ejecutiva (FRSBEe), y proporciona también una puntuación global (FRSBEt). Las respuestas se efectúan en una escala tipo Likert, de acuerdo con el instrumento original. Los items 1-32 representan déficits en la función prefrontal, y altas puntuaciones indican gran disfunción $(1=$ Casi nunca, $2=$ Pocas veces, $3=$ Algunas veces, $4=$ Frecuentemente, 5 = Casi siempre). Los ítems 33-46, exploran buen funcionamiento ejecutivo y las puntuaciones se requieren de forma inversa. El FRSBE ha mostrado elevada consistencia interna en sus escalas tanto en muestras clínicas como en población normal. Ha sido validado en poblaciones con patologías neuropsicológicas que incluyen disfunción prefrontal y subcortical. A pesar de tratarse de un autoinforme subjetivo, ha mostrado elevada validez diagnóstica en muestras clínicas y frente a pruebas de ejecución neuropsicológica. Recientemente se ha validado la versión española del cuestionario (Pedrero, Ruiz Sánchez de León, Llanero, Rojo, Olivar y Puerta, en prensa), que será la empleada en el presente trabajo.

Dickman's Impulsivity Inventory, Short Version (DIIshort; Dickman, 1990) consistente en dos escalas binarias de evaluación de aspectos relativos a impulsividad funcional (11 items) y disfuncional (12 items). La impulsividad funcional representa un rasgo que se traduce en buen rendimiento cuando las tareas requieren respuestas rápidas. La impulsividad disfuncional, en cambio, se refiere a la tendencia a involucrarse en procesamiento rápido e irreflexivo, y frecuentemente erróneo. En el presente estudio se ha utilizado una versión previamente validada en español (Chico et al., 2003).

\section{Análisis de datos}

Se efectuaron en primer lugar pruebas de consistencia interna sobre la prueba completa y sobre sus elementos. En segundo lugar se procedió a un estudio de la validez del test, comenzando por su estructura factorial a partir de un análisis exploratorio sobre la submuestra no clínica, aplicando estimadores de consistencia interna ( $q$ de Carmines) sobre los componentes principales sin rotar. Posteriormente, se diseñó una prueba confirmatoria sobre la muestra clínica y se compararon los resultados con los obtenidos en trabajos precedentes. Se estudió a continuación la dimensionalidad de la prueba, para, en conjunción con los datos anteriores, formular un modelo dimensional del cuestionario. Finalmente, se valoraron pruebas de validez convergente y discriminante y se estimaron, mediante modelos multivariantes que controlaran los desequilibrios entre las muestras en variables intervinientes, las diferencias principales entre las submuestras clínica y no clínica, así como las diferencias por sexo aparecidas en el estudio, de cara a explorar la aplicabilidad de la prueba en diversas muestras.

\section{RESULTADOS}

\section{Consistencia interna}

El alfa de Cronbach fue de 0,91 (0,79 en la submuestra no clínica y 0,92 en la submuestra clínica). Los estadísticos de los ítems se exponen en la Tabla 2. El ítem 15 mostró una no correlación con el cuestionario en su globalidad y de ser excluido se incrementaría la consistencia interna de la escala. La correlación intraclase resultó adecuada $(p<0,001)$, variando entre 0,30 y 0,94 .

Tabla 2. Estadísticos de los ítems de la versión española del DEX (DEX-Sp)

\begin{tabular}{lcccccc}
\hline & Media & D.t. & $\begin{array}{c}\text { Media sin } \\
\text { el item }\end{array}$ & $\begin{array}{c}\text { Varianza sin } \\
\text { el item }\end{array}$ & $\begin{array}{c}\text { Correlación } \\
\text { item-total } \\
\text { corregida }\end{array}$ & $\begin{array}{c}\text { sin el } \\
\text { item }\end{array}$ \\
\hline İtem 1 & 1,71 & 0,86 & 42,29 & 162,55 & 0,56 & 0,91 \\
İtem 2 & 2,42 & 1,06 & 41,59 & 157,19 & 0,65 & 0,90 \\
İtem 3 & 1,34 & 0,72 & 42,66 & 165,80 & 0,50 & 0,91 \\
İtem 4 & 2,02 & 1,09 & 41,98 & 157,51 & 0,62 & 0,91 \\
İtem 5 & 2,35 & 1,04 & 41,65 & 156,78 & 0,68 & 0,90 \\
İtem 6 & 1,78 & 1,07 & 42,23 & 156,15 & 0,68 & 0,90 \\
İtem 7 & 2,16 & 1,31 & 41,84 & 150,93 & 0,71 & 0,90 \\
İtem 8 & 2,34 & 1,28 & 41,66 & 151,42 & 0,72 & 0,90 \\
İtem 9 & 1,55 & 0,82 & 42,45 & 164,84 & 0,48 & 0,91 \\
Item 10 & 2,12 & 1,05 & 41,89 & 157,98 & 0,63 & 0,90 \\
İtem 11 & 2,45 & 1,20 & 41,56 & 162,68 & 0,38 & 0,91 \\
İtem 12 & 2,30 & 0,99 & 41,71 & 161,11 & 0,54 & 0,91 \\
İem 13 & 2,00 & 1,02 & 42,00 & 162,81 & 0,45 & 0,91 \\
İtem 14 & 2,17 & 1,06 & 41,83 & 160,62 & 0,52 & 0,91 \\
İtem 15 & 2,98 & 1,24 & 41,02 & 172,52 & 0,05 & 0,92 \\
İtem 16 & 2,30 & 1,15 & 41,70 & 156,77 & 0,61 & 0,91 \\
İtem 17 & 2,39 & 1,11 & 41,62 & 155,96 & 0,67 & 0,90 \\
İtem 18 & 2,59 & 1,16 & 41,42 & 154,10 & 0,70 & 0,90 \\
Item 19 & 2,54 & 1,17 & 41,47 & 155,08 & 0,66 & 0,90 \\
Item 20 & 2,50 & 1,15 & 41,51 & 162,06 & 0,42 & 0,91 \\
\hline
\end{tabular}

Se efectuó un análisis de componentes principales en la submuestra no clínica, para determinar los factores claves del síndrome disejecutivo medido por el DEX. La medida de adecuación muestral fue de 0,73 y la prueba de esfericidad de Barlett fue también significativa $(2=581,6 ; p<0,0001)$, indicando la viabilidad de criterios para el análisis factorial (Hair, Anderson, Tatham y Black, 1995). Aparecieron siete factores con autovalores mayores de $1(4,44[q=0,82], 1,88$ $[q=0,49], 1,51[q=0,36], 1,32[q=0,26], 1,11[q=0,10]$, $1,07[q=0,07]$ y $1,03[q=0,03])$ que daban cuenta del $62 \%$ de la varianza total. El Scree-test (Cattell, 1978) sugirió que la mejor solución era la de cinco. Esta solución explicaría el $51,3 \%$ de la varianza total. Se realizó a continuación una rotación ortogonal Varimax sobre esta solución de cinco factores. La Tabla 3 muestra esta solución junto con las cargas de cada ítem en cada factor y el porcentaje de varianza explicada. 
Tabla 3. Análisis de componentes principales con rotación Varimax de los ítems del DEX-Sp

\begin{tabular}{|c|c|c|c|c|c|c|}
\hline \multirow[b]{2}{*}{ İtem } & \multirow[b]{2}{*}{ Descripción } & \multicolumn{5}{|c|}{ Componentes } \\
\hline & & 1 & 2 & 3 & 4 & 5 \\
\hline 4 & Problemas de planificación & 0,72 & 0,15 & $-0,04$ & $-0,09$ & 0,05 \\
\hline 8 & Apatía & 0,72 & 0,27 & $-0,12$ & 0,22 & 0,05 \\
\hline 19 & $\begin{array}{l}\text { Pobre habilidad en la toma de } \\
\text { decisiones }\end{array}$ & 0,61 & 0,02 & 0,19 & 0,20 & $-0,14$ \\
\hline 11 & Respuestas afectivas superficiales & 0,60 & 0,04 & 0,32 & 0,01 & $-0,09$ \\
\hline 6 & $\begin{array}{l}\text { Problemas de secuenciación } \\
\text { temporal }\end{array}$ & 0,47 & $-0,09$ & 0,44 & 0,23 & 0,18 \\
\hline 18 & Distractibilidad & 0,46 & 0,37 & 0,17 & $-0,02$ & 0,06 \\
\hline 12 & Agresión & 0,11 & 0,73 & 0,19 & $-0,17$ & $-0,19$ \\
\hline 5 & Euforia & 0,21 & 0,70 & $-0,01$ & $-0,04$ & 0,19 \\
\hline 9 & Desinhibición & $-0,04$ & 0,63 & 0,02 & 0,38 & $-0,03$ \\
\hline 7 & Falta de insight & 0,30 & 0,47 & 0,11 & 0,21 & 0,07 \\
\hline 14 & Perseveración & 0,13 & 0,13 & 0,79 & 0,10 & $-0,04$ \\
\hline 13 & Falta de interés & 0,15 & 0,04 & 0,71 & $-0,02$ & 0,13 \\
\hline 2 & Impulsividad & 0,00 & 0,38 & 0,44 & 0,16 & 0,07 \\
\hline 3 & Fabulación & $-0,07$ & $-0,09$ & $-0,10$ & 0,81 & 0,17 \\
\hline 1 & $\begin{array}{l}\text { Problemas en el pensamiento } \\
\text { abstracto }\end{array}$ & 0,35 & 0,05 & 0,27 & 0,46 & $-0,18$ \\
\hline 17 & $\begin{array}{l}\text { Disociación entre conocimiento y } \\
\text { respuesta }\end{array}$ & 0,15 & 0,27 & 0,20 & 0,44 & 0,06 \\
\hline 10 & $\begin{array}{l}\text { Dificultades en el control de los } \\
\text { impulsos }\end{array}$ & 0,24 & 0,15 & 0,28 & 0,42 & 0,16 \\
\hline 15 & Inquietud & $-0,16$ & $-0,02$ & 0,06 & 0,07 & 0,73 \\
\hline 20 & $\begin{array}{l}\text { Falta de interés por las reglas } \\
\text { sociales }\end{array}$ & 0,16 & 0,02 & 0,02 & 0,06 & 0,67 \\
\hline 16 & $\begin{array}{l}\text { Falta de habilidad para inhibir } \\
\text { respuestas }\end{array}$ & $-0,11$ & 0,23 & 0,48 & 0,14 & 0,53 \\
\hline Porc & entaje de la varianza total explicado & 13,3 & 11,0 & 10,9 & 8,4 & 7,7 \\
\hline
\end{tabular}

NOTA: Las cargas principales en cada factor se han resaltado en negrita.

A continuación se realizó un análisis factorial confirmatorio en la submuestra clínica, mediante el método de estimación de máxima probabilidad (Hu y Bentler, 1995). El estadístico 2 muestra una adecuada bondad de ajuste del modelo, aunque también una alta sensibilidad al tamaño muestral. Por ello, se usa frecuentemente la discrepancia muestral mínima dividida por los grados de libertad (CMIN/ DF; Wheaton, Muthén, Alwin y Summers, 1977), siendo los resultados menores de 2 indicadores de excelente bondad de ajuste y entre 2 y 3 de niveles aceptables (Carmines y Mclver, 1981). El índice comparativo de ajuste (CFI; Bentler, 1990), el índice de ajuste normativo (NFI; Bentler y Bonett, 1980) y el indice incremental de ajuste (IFI; Bollen, 1989) pueden oscilar entre 0 (falta de ajuste) y 1 (ajuste óptimo). El índice parsimonioso de ajuste (PCFI; James et al., 1982) se ha propuesto como un indicador más preciso que los precedentes, puesto que es capaz de tomar en consideración la parsimonia de los modelos en el juicio de ajuste. Puntuaciones entre 0,5 y 0,7 resultan aceptables. La raíz cuadrada del error de aproximación (RMSEA; Browne y Cudeck, 1993) se considera óptima cuando sus valores son inferiores a 0,5 (Hu y Bentler, 1995).

Las soluciones de 4 y 5 factores obtenidas en la submuestra no clínica se sometieron a contrastación en la submuestra clínica. Además, se contrastaron otras estructuras de items propuestas por diversos autores (Amieva et al., 2003; Burgess et al., 1998; Chan et al., 2001; Chaytor et al., 2007; Mooney et al., 2006). La Tabla 4 muestra las medidas de ajuste de cada modelo contrastadas en la submuestra clínica. Los resultados muestran que ninguno de los modelos ofrece mejoras significativas sobre los demás. El modelo de Mooney es el único que muestra un valor por debajo de 2 en la discrepancia muestral mínima dividida por los grados de libertad (CMIN/DF). Todos los modelos muestran un buen ajuste en los indices generales (NFI, IFI, CFI) pero impreciso en las medidas más específicas (PCFI y RMSEA).

Tabla 4. Índices de bondad de ajuste de las soluciones de 4 y 5 factores del DEX-Sp, y de otras estructuras propuestas por otros autores en trabajos previos

\begin{tabular}{|c|c|c|c|c|c|c|c|c|c|}
\hline & \multicolumn{9}{|c|}{ Medidas de ajuste } \\
\hline & 2 & $\mathrm{GL}$ & NPAR & CMINDF & $N F I$ & $\mathrm{IFI}$ & $\mathrm{CFI}$ & PCFI & RMSEA \\
\hline Modelo independiente & 5718,76 & 210 & 20 & 27,23 & 0,00 & 0,00 & 0,00 & 0,00 & 0,46 \\
\hline Modelo de 4 factores & 334,62 & 164 & 66 & 2,04 & 0,94 & 0,97 & 0,97 & 0,76 & 0,09 \\
\hline Modelo de 5 factores & 332,94 & 160 & 70 & 2,08 & 0,94 & 0,97 & 0,97 & 0,74 & 0,09 \\
\hline${ }^{*}$ Chan & 286,67 & 125 & 64 & 2,29 & 0,94 & 0,97 & 0,97 & 0,71 & 0,10 \\
\hline${ }^{*}$ Amieva & 346,27 & 160 & 70 & 2,16 & 0,94 & 0,97 & 0,97 & 0,74 & 0,10 \\
\hline${ }^{* * *}$ Mooney & 282,02 & 146 & 63 & 1,93 & 0,95 & 0,97 & 0,97 & 0,75 & 0,09 \\
\hline${ }^{* * * *}$ Burgess & 330,84 & 160 & 70 & 2,07 & 0,94 & 0,97 & 0,97 & 0,74 & 0,09 \\
\hline ***** Chaytor & 332,51 & 160 & 70 & 2,08 & 0,94 & 0,97 & 0,97 & 0,74 & 0,09 \\
\hline
\end{tabular}

$\mathrm{GL}=$ Grados de libertad; NPAR=Número de parámetros; $\mathrm{CMIN/DF=}{ }^{2}$ relativa; $\mathrm{NFI}=$ İndice de ajuste normativo; IFI= İndice de ajuste incremental; $\mathrm{CFI}=$ İndice de ajuste comparativo; PCFI= İndice de ajuste parsimonioso; RMSEA = Raiz cuadrada del error de aproximación.

*Chan, 2001; **Amieva, Phillips y Della Sala, 2003; ***Mooney, Walmsley y McFarland, 2006; **** Burgess, Alderman, Evans, Emslie y Wilson, 1998; **** Chaytor y Schmitter-Edgecombe, 2007.

No aparecen razones suficientes para optar por un modelo particular a partir de sus indicadores de ajuste. Estudiando trabajos precedentes (Tabla 5) encontramos que se aceptan tanto las soluciones de 4 como las de 5 factores. Además, los ítems son asignados a factores con diferentes denominaciones en cada uno de los modelos. Por ejemplo, el ítem 1 puede incluirse en factores denominados, según el trabajo, Resistencia, Afrontamiento de Interferencias, Memoria Ejecutiva, Solución de Problemas Abstractos e Inhibición. La consistencia interna de cada factor, en las soluciones de 4 y de 5, se muestran en la Tabla 6 . 
Tabla 5. Soluciones factoriales y denominación de los componentes encontrados en trabajos previos y la solución de 5 factores del presente estudio

\begin{tabular}{|c|c|c|c|c|c|c|}
\hline & (1) Burgess & (2) Chan & (3) Amieva & (4) Mooney & (5) Chaytor & Presente estudio \\
\hline Numero de factores & 5 & 5 & 5 & 4 & 5 & 5 \\
\hline Varianza explicada & $67 \%$ & $63 \%$ & $76 \%$ & $54 \%$ & $72 \%$ & $51 \%$ \\
\hline İtem 01 & Inhibición & Resistencia interna & Superar interferencias & $\begin{array}{c}\text { Solución de problemas } \\
\text { abstractos }\end{array}$ & Memoria ejecutiva & 4 \\
\hline İtem 02 & Inhibición & Intencionalidad & Intencionalidad & Inhibición & Inhibición & 3 \\
\hline İtem 03 & Memoria ejecutiva & Resistencia interna & Inhibición & $\begin{array}{c}\text { Solución de problemas } \\
\text { abstractos }\end{array}$ & Falta de conciencia & 4 \\
\hline İtem 04 & Intencionalidad & Intencionalidad & Planificación & Social regulation & Intencionalidad & 1 \\
\hline İtem 05 & Positive affect & Inhibición & Inhibición & Inhibición & Intencionalidad & 2 \\
\hline İtem 06 & Memoria ejecutiva & (a) & Superar interferencias & $\begin{array}{c}\text { Solución de problemas } \\
\text { abstractos }\end{array}$ & Memoria ejecutiva & 1 \\
\hline İtem 07 & Intencionalidad & $\begin{array}{c}\text { Disociación pensamien- } \\
\text { to-acción }\end{array}$ & Planificación & Regulación social & Falta de conciencia & 2 \\
\hline İtem 08 & Afecto negativo & $\begin{array}{c}\text { Disociación pensamien- } \\
\text { to-acción }\end{array}$ & Intencionalidad & Regulación social & Intencionalidad & 1 \\
\hline İtem 09 & Inhibición & Inhibición & Regulación social & $\begin{array}{c}\text { Solución de problemas } \\
\text { abstractos }\end{array}$ & Inhibición & 2 \\
\hline İtem 10 & Afecto positivo & (a) & Intencionalidad & (a) & Intencionalidad & 4 \\
\hline İtem 11 & Afecto negativo & $\begin{array}{c}\text { Disociación pensamien- } \\
\text { to-acción }\end{array}$ & Superar interferencias & Regulación social & Inhibición & 1 \\
\hline İtem 12 & Afecto positivo & Regulación social & Regulación social & Inhibición & Inhibición & 2 \\
\hline İtem 13 & Inhibición & Inhibición & Regulación social & Inhibición & Inhibición & 3 \\
\hline İtem 14 & Memoria ejecutiva & Resistencia interna & Inhibición & Inhibición & Memoria ejecutiva & 3 \\
\hline İtem 15 & Inhibición & Inhibición & Superar interferencias & Inhibición & $\begin{array}{c}\text { Agitación/ } \\
\text { Hiperactividad }\end{array}$ & 5 \\
\hline İtem 16 & Inhibición & Inhibición & Regulación social & Regulación social & $\begin{array}{c}\text { Agitación/ } \\
\text { Hiperactividad }\end{array}$ & 5 \\
\hline İtem 17 & Intencionalidad & $\begin{array}{c}\text { Disociación pensamien- } \\
\text { to-acción }\end{array}$ & Planificación & Intencionalidad & Falta de conciencia & 4 \\
\hline İtem 18 & Intencionalidad & Intencionalidad & Superar interferencias & Intencionalidad & Memoria ejecutiva & 1 \\
\hline İtem 19 & Intencionalidad & Intencionalidad & Planificación & Intencionalidad & Intencionalidad & 1 \\
\hline İtem 20 & Inhibición & Regulación social & Intencionalidad & Regulación social & Inhibición & 5 \\
\hline N & 308 & 93 & 20 & 342 & 46 & 258 \\
\hline
\end{tabular}

(1) Burgess, Alderman, Evans, Emslie y Wilson, 1998; (2) Chan, 2001; (3) Amieva, Phillips y Della Sala, 2003; (4) Mooney, Walmsley y McFarland, 2006 ; (5) Chaytor y Schmitter-Edgecombe, 2007. (a) İtem no incluido en ningún factor.

Tabla 6. Consistencia interna ( de Cronbach) de las soluciones de 4 y 5 factores

\begin{tabular}{cccc}
\hline & Muestra total & $\begin{array}{c}\text { Submuestra no } \\
\text { clínica }\end{array}$ & Submuestra clinica \\
\hline F1_4 & 0,85 & 0,74 & 0,84 \\
F2_4 & 0,75 & 0,64 & 0,74 \\
F3_4 & 0,70 & 0,63 & 0,68 \\
F4_4 & 0,57 & 0,42 & 0,60 \\
\hline F1_5 & & & 0,83 \\
F2_5 & 0,84 & 0,73 & 0,74 \\
F3_5 & 0,75 & 0,64 & 0,58 \\
F4_5 & 0,62 & 0,59 & 0,71 \\
F5_5 & 0,72 & 0,48 & 0,39 \\
\hline
\end{tabular}

Fx_4= Componentes de la solución de 4 factores. Fx_5= Componentes de la solución de 5 factores.

\section{Dimensionalidad}

Cuando exploramos la posible unidimensionalidad de la escala no encontramos suficientes indicadores para aceptarla. El coeficiente theta de Carmines (Carmines y Zeller, 1979) del primer factor sin rotar es notablemente mayor que el del resto de componentes $(q=0,82)$, pero no se aproxima suficientemente a la unidad (tampoco la eliminación del problemático ítem 15 mejoraría ni en una décima este valor). Por otra parte, aunque la mayor parte de los ítems presentan su carga principal en este factor, no todos se comportan del mismo modo: 7 ítems cargan más en alguno de los otros 6 factores. Tampoco se cumple la regla propuesta por diversos autores (Gorsuch, 1983; Hattie, 1984, 1985) que consideran que el primer factor debe dar cuenta de 4 veces más varianza que el segundo para poder afirmar 
la unidimensionalidad de la escala; en nuestra muestra la relación es de $2,4(4,44 / 1,88)$.

La Figura 1 muestra el resultado del escalamiento bidimensional de los items del DEX. Se efectuó un escalamiento clásico (ALSCAL) y la solución resultante mostró una adecuada bondad de ajuste (S-Stress 0,07; $\mathrm{RSO}=0,98)$. El análisis del mapa resultante revela que todos los ítems aparecen distribuidos en un espacio común y en gran proximidad, excepto el ítem 15, situado en una región muy alejada del resto. Diferentes soluciones ensayadas sitúan consistentemente al ítem 15 en una posición marginal.

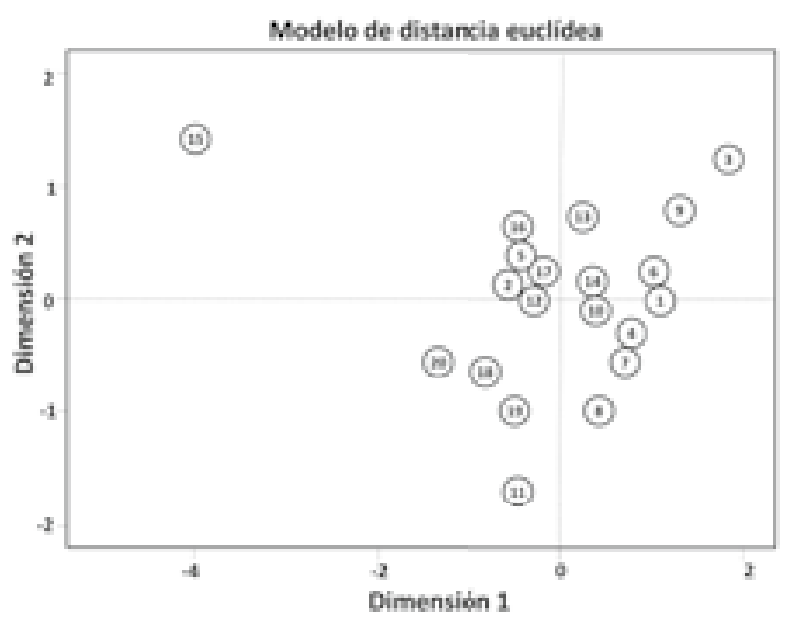

Figura 1. Escalamiento bidimensional ALSCAL de los ítems del DEX-Sp

\section{Modelo estructural}

Las estructuras derivadas de las soluciones de 4 y 5 factores se muestran en la Figura 2, así como las covarianzas entre los factores y sus respectivos ítems. Los factores 4 y 5 en la solución de 5 factores aparecen juntos en la estructura de 4 factores, con excepción del ítem 1 (que carga en el factor 1 ) y el ítem 16 (que carga en el factor 3 ). El resto de factores mantienen la misma estructura en las dos soluciones.

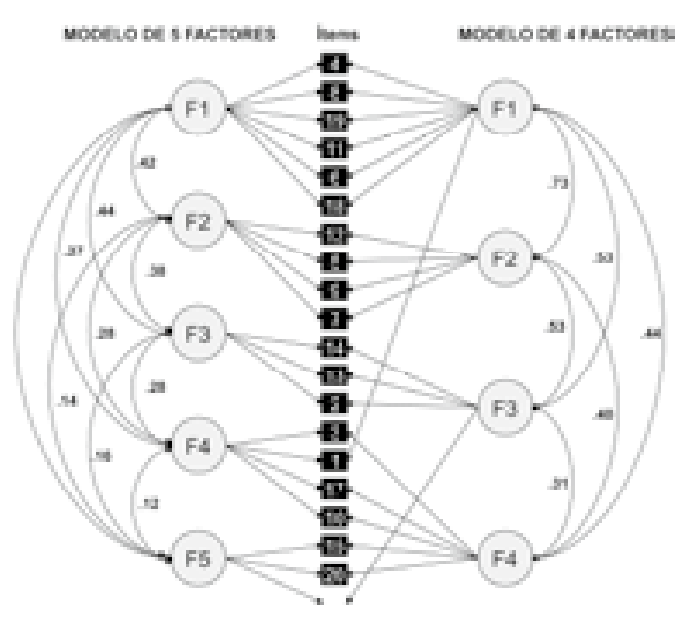

Figura 2. Estructura derivada de las soluciones de 4 y 5 factores de los ítems del DEX-Sp

\section{Validez convergente}

La Tabla 7 muestra las correlaciones entre factores (en las soluciones de 4 y 5) y entre las puntuaciones totales del DEX y las escalas del FrSBe. Sexo, edad y nivel académico fueron controlados para evitar sesgos. Cada correlación muestra un alto nivel de significación estadística $(p<0,00001)$ y en la mayor parte de los casos un tamaño del efecto sustancial. Estos resultados apoyan la convergencia de ambos cuestionarios en la medida de constructos relacionados.

Tabla 7. Correlaciones parciales (controlando sexo, edad y nivel de estudios) entre los componentes del DEX-Sp (soluciones de 4 y 5 factores) y los componentes de la Frontal Behavior Scale (FrSBe) y el Dickman Impulsivity Inventory-Short Versión (DII-Short)

\begin{tabular}{cccccc}
\hline & \multicolumn{4}{c}{ FrSBe } & DII-short \\
\cline { 2 - 6 } DEX & $\begin{array}{c}\text { Disfunción } \\
\text { Ejecutiva }\end{array}$ & Apatia & Desinhibición & Disfuncional & Funcional \\
\hline F1_5 & $0,68^{* * *}$ & $0,64^{* * *}$ & $0,54^{* * *}$ & $0,57^{* * *}$ & $-0,21$ \\
F2_5 & $0,65^{* * *}$ & $0,51^{* * *}$ & $0,63^{* * *}$ & $0,57^{* * *}$ & $-0,04$ \\
F3_5 & $0,60^{* * *}$ & $0,50^{* * *}$ & $0,58^{* * *}$ & $0,63^{* * *}$ & 0,11 \\
F4_5 & $0,62^{* * *}$ & $0,51^{* * *}$ & $0,53^{* * *}$ & $0,58^{* * *}$ & 0,10 \\
F5_5 & $0,33^{* * *}$ & 0,16 & $0,36^{* * *}$ & $0,39^{* * *}$ & $0,28^{* * *}$ \\
F1_4 & $0,68^{* * *}$ & $0,64^{* * *}$ & $0,54^{* * *}$ & $0,58^{* * *}$ & $-0,20$ \\
F2_4 & $0,65^{* * *}$ & $0,51^{* * *}$ & $0,63^{* * *}$ & $0,57^{* * *}$ & $-0,04$ \\
F3_4 & $0,61^{* * *}$ & $0,48^{* * *}$ & $0,59^{* * *}$ & $0,66^{* * *}$ & 0,18 \\
F4_4 & $0,51^{* * *}$ & $0,37^{* * *}$ & $0,49^{* * *}$ & $0,49^{* * *}$ & 0,23 \\
\hline DEX Total & $0,72^{* * *}$ & $0,60^{* * *}$ & $0,64^{* * *}$ & $0,70^{* * *}$ & 0,00 \\
\hline
\end{tabular}

NOTA: *** $p<0,001 ; F x \_4=$ Componentes de la solución de 4 factores; Fx 5= Componentes de la solución de 5 factores.

\section{Validez discriminante}

Se exploraron las relaciones entre los componentes del DEX y las escalas del DII-Short. Este cuestionario proporciona 2 medidas independientes de impulsividad. La Impulsividad Disfuncional debería correlacionar con disfunción ejecutiva, en tanto que la Impulsividad Funcional no debería hacerlo, en la medida que está relacionada con el procesamiento rápido de la información y la conducta orientada a metas. Como se muestra en la Tabla 7, esta hipótesis se confirma plenamente, salvo en el caso del $5^{\circ}$ factor de la solución de 5 factores. Este hecho puede tener dos explicaciones: en primer lugar, se trata de un factor que mide hiperkinesia/impulsividad, especialmente relacionado con el cuestionario de Dickman; en segundo lugar, este factor puede no estar informando sobre verdaderos síntomas disfuncionales y ello puede estar relacionado con la localización marginal del ítem 15 (Figura 1). Probablemente, el ítem 15 evalúa actividad funcional $y$, a causa de ello, aparece aislado en el escalamiento dimensional. 


\section{Diferencias de género, edad y nivel académico}

No se encontraron diferencias estadísticas por sexo en la submuestra no clínica en la puntuación total del DEX (prueba de Mann-Whitney, $Z=0,75 ; p=0,45$ ). No obstante las mujeres de la submuestra clínica puntuaron significativamente más alto que los varones $(Z=2,2 ; p<0,05)$. La comparación por sexo entre ambas submuestras ofrece amplias y sólidas diferencias, tanto para los varones $(Z=4,8$; $p<0,001)$ como para las mujeres $(Z=5,3 ; p<0,001)$.

Controlando los efectos del sexo y la edad, las puntuaciones totales del DEX muestran correlaciones negativas con el nivel académico alcanzado $(r=-0,26$; $p<0,001)$. Por el contrario, controlando el sexo y el nivel académico, no aparece correlación entre las puntuaciones del DEX y la edad $(r=0,09 ; p=0,17)$.

\section{Diferencias entre submuestras clínica y no clínica}

Finalmente, se analizaron las diferencias entre sujetos de las submuestras clínica y no clínica en los componentes del DEX y su puntuación global. En primer lugar, se efectuó un test de Levene, que no garantizó la homogeneidad de las varianzas en 8 de 10 comparaciones. La prueba robusta de Brown-Forsythe mostró diferencias estadísticas en todas las medias $(p<0,0001)$. Un análisis multivariado, controlando el sexo, la edad y el nivel de estudios, se muestra en la Tabla 8. Se aplicó el estadístico eta al cuadrado parcial $\left(h_{p}^{2}\right)$ como estimador del tamaño del efecto de las diferencias. Aparecieron diferencias significativas y moderado tamaño del efecto entre ambas submuestras en todas las dimensiones del DEX y su puntuación total. Los adictos puntúan más en todas las comparaciones.

Tabla 8. Comparación multivariada de los componentes del DEX entre las submuestras clínica y no clínica, controlando sexo, edad y nivel de estudios.

\begin{tabular}{cccccccc}
\hline \multicolumn{7}{c}{ No clínica } & \multicolumn{7}{c}{ Clinica (adictos) } \\
\hline $\begin{array}{c}\text { Factores del } \\
\text { DEX-Sp }\end{array}$ & Media & D.t. & Media & D.t. & F & Sig. & $h^{2}$ \\
\hline F1_5 & 11,19 & 3,47 & 16,31 & 5,45 & 24,37 & $p<, 0001$ & 0,28 \\
F2_5 & 6,88 & 2,18 & 9,89 & 3,37 & 22,68 & $p<, 0001$ & 0,26 \\
F3_5 & 5,92 & 2,06 & 7,29 & 2,45 & 9,00 & $p<, 0001$ & 0,13 \\
F4_5 & 6,31 & 1,70 & 8,83 & 3,11 & 21,30 & $p<, 0001$ & 0,25 \\
F5_5 & 7,21 & 2,27 & 8,36 & 2,43 & 4,14 & $p<, 01$ & 0,06 \\
F1_4 & 12,69 & 3,74 & 18,24 & 6,10 & 23,95 & $p<, 0001$ & 0,28 \\
F2_4 & 6,88 & 2,18 & 9,89 & 3,37 & 22,68 & $p<, 0001$ & 0,26 \\
F3_4 & 7,74 & 2,58 & 10,09 & 3,17 & 12,83 & $p<, 0001$ & 0,17 \\
F4_4 & 10,21 & 2,45 & 12,46 & 3,55 & 10,38 & $p<, 0001$ & 0,14 \\
\hline DEX_Total & 37,52 & 7,78 & 50,69 & 14,39 & 25,55 & $p<, 0001$ & 0,50 \\
\hline
\end{tabular}

$\mathrm{h}^{2}=$ Eta al cuadrado parcial como estimador del tamaño del efecto; Fx_4=Componentes de la solución de 4 factores; Fx_5= Componentes de la solución de 5 factores.

\section{DISCUSIÓN}

El estudio psicométrico de la versión española del DEX apunta a su fiabilidad como escala de medida. Sin embargo, el análisis factorial no ofrece una solución clara en cuanto a su estructura. Éste es un hecho común en todos los estudios de la estructura del DEX, tanto en su versión auto como en la versión heteroadministrada. Diversas soluciones de 4 y 5 factores han sido propuestas pero con amplias discrepancias en cuanto a la asignación de items a los factores. En estudios con N > 200, observamos que, mientras Burgess et al. (1998) proponen 5 factores, Mooney et al. (2006) proponen sólo 4. En ambos casos, la denominación de los factores y los ítems asignados a cada uno de ellos sólo coincide en 6 de los 20 ítems. La variabilidad estructural del DEX puede estar originada por el pequeño número de ítems y la dificultad para dar cuenta de la globalidad de los síntomas disejecutivos en las actividades de la vida cotidiana.

El item 15 parece medir un constructo diferente al estimado por el resto. Algunos autores encuentran problemas similares y recomiendan la exclusión de los ítems conflictivos. Por ejemplo, Mooney et al. (2006) excluyen el ítem 10, y Chan et al. (2001) hacen lo mismo con los items 6 y 10. En nuestro trabajo, el ítem 15 parece evaluar una dimensión hiperkinética probablemente asociada a disfunción ejecutiva, pero no relacionada con el resto de síntomas cognitivos evaluados por el resto de los ítems.

Nuestra solución de 5 factores muestra que el primero de ellos se refiere a problemas en habilidades de planificación y su relación con resolución de problemas y toma de decisiones, junto con apatía y sintomas de falta de activación. El segundo factor agrupa síntomas de desinhibición, euforia, agresión y falta de insight y conciencia social. Sin embargo, el patrón de correlaciones de ambos factores con otras escalas (FrSBe y DII-Short, en este trabajo) es similar, lo que viene a sugerir que no existen diferencias significativas en su contenido. El tercer factor agrupa sintomas de impulsividad y perseveración. El cuarto factor agrupa fabulación y problemas de persistencia, en el sentido de falta de mantenimiento de los planes. El quinto factor parece relacionarse con falta de habilidad para inhibir respuestas, inquietud e hiperkinesia, así como falta de implicación con las reglas sociales. A pesar de que el primer factor se relaciona tradicionalmente con disfunción dorsolateral y medial superior y el segundo con disfunción orbitofrontal medial inferior, no se han informado lesiones focales en los participantes. No están claras las hipotéticas localizaciones para los factores 3,4 y 5 . En definitiva, no hay razones para establecer relaciones anatomofuncionales ni daño focal que pueda ser explicado por los resultados proporcionados por los adictos.

Hemos explorado también la posible unidimensionalidad del DEX, no encontrando argumentos suficientes para considerarla. No obstante, a tenor de la confusa identificación de los factores (de manera similar a la observada en otros estudios), y, desde una consideración meramente clínica, parece razonable considerar al DEX como un índice univariado de sintomatología disejecutiva, como prueba de cribado inicial, que dé paso a pruebas más específicas (p.e., la Escala 
de Comportamiento Frontal, Pedrero et al., en prensa) y a una evaluación de rendimiento neuropsicológico que permita identificar de forma más precisa la naturaleza de los déficits cognitivos sugeridos en el cribado.

Nuestros datos apoyan el hecho de que el DEX y la Escala de Comportamiento Frontal, una escala propuesta para medir variables similares, muestran convergencia de medidas (correlación $0,60<r<0,72$ para las 3 escalas del FrSBe) y que ambos instrumentos evalúan sintomas similares. También aparece convergencia con la medida de la impulsividad disfuncional del DII-Short (correlación con el DEX $r=0,7$ ), que es un componente conocido del sindrome disejecutivo. No se encuentra relación con la impulsividad funcional, que Dickman adscribe al procesamiento rápido de la información y la toma de decisiones orientada a objetivos funcionales.

En conclusión, los datos sugieren que la versión española del DEX (DEX-Sp, Apéndice 1) es una medida útil de evaluación general de sintomas del síndrome disejecutivo. La disfunción cerebral en adictos puede ser mucho más sutil (Goldstein, Leskovjan, Hoff, Hitzemann, Bashan, Calza et al., 2004) y reversible (Selby y Azrin, 1998) que en el daño cerebral de otras etiologías. No hay que perder de vista las críticas a la validez ecológica de este tipo de medidas (Wilson et al, 1996). No obstante, la anosognosia asociada al síndrome disejecutivo en el daño cerebral por otras causas puede ser más severo que en adictos que inician tratamiento: éstos lo hacen desde la decisión voluntaria de reducir el deterioro provocado por el abuso de las sustancias, lo cual, de hecho, implica cierta conciencia de problema.

En definitiva, podemos considerar a la versión española del DEX (DEX-Sp) como un instrumento fiable y válido de cribado inicial con capacidad para detectar deterioros cognitivos asociados al abuso de sustancias. Puede ser recomendado para desarrollar una evaluación en el tratamiento de las adicciones, para establecer un proyecto de rehabilitación neurocognitiva y para proponer hipótesis alternativas a la pretendida comorbilidad psiquiátrica. Se propone la inclusión del DEX-Sp en la batería de evaluación inicial de los adictos incluidos en tratamiento, dada su facilidad de cumplimentación, brevedad y bajo coste, como paso preliminar y cribado para la ejecución de medidas psicopatológicas, neuropsicológicas y neurológicas más complejas.

\section{Limitaciones}

El tamaño de la muestra (131 adictos) cumple los criterios mínimos expuestos por algunos autores acerca del ratio de sujetos por item en el análisis factorial (5:1 en el más liberal; Nunnally, 1978; Kline, 1994). Sin embargo, esta pequeña muestra puede resultar en una solución factorial inestable. No obstante, ninguno de los trabajos revisados alcanza los ratios propuestos como más exigentes (15:1 a 30:1; Pedhazur, 1997), y aquellos que utilizan muestras más grandes (Burgess et al., 1998 y Mooney et al., 2006) tampoco encuentran resultados similares en la estructura factorial.
Otra importante limitación es la que se refiere a las amplias diferencias en la composición de las submuestras clínica y no clínica. La submuestra no clínica está compuesta mayoritariamente por mujeres; por el contrario, la submuestra clínica refleja la preponderancia habitual de varones en las poblaciones de adictos en tratamiento. Adicionalmente, la edad y el nivel académico presentan amplias diferencias entre ambos subgrupos. Éste es uno de los problemas habitualmente encontrados cuando se utiliza el método de muestreo "en bola de nieve". Una solución derivada mayoritariamente de mujeres puede no resultar ideal para capturar elevaciones reales en sintomatología disejecutiva en varones adictos. Sin embargo, éste es un problema compartido por los estudios similares, que muestran ratios superiores a 1,0 en el cociente mujeres/ hombres en población no clínica -por ejemplo, Chan (2001; ratio 64/29), Wang et al. (2006; ratio 75/49) o no declarado (Alderman et al., 2001; Burgess et al., 1998), mientras que las muestras clínicas están habitualmente compuestas por más varones (Wood et al., 2006, ratio 18/41; Mathias, 2003, ratio 13/17; Chaytor et al., 2006-2007, ratio 20/26). Los autores del presente trabajo han dado cuenta estadisticamente de estas diferencias, usando análisis multivariados y controlando las variables intervinientes. Se requeririan muestras más amplias y equilibradas para obtener conclusiones más precisas.

Los autores declaran que no existen conflictos de intereses, ni fuentes de financiación externa para esta investigación.

\section{REFERENCIAS}

Aharonovich, E., Hasin, D. S., Brooks, A. C., Liu, X., Bisaga, A. y Nunes, E. V. (2006). Cognitive deficits predict low treatment retention in cocaine dependent patients. Drug and Alcohol Dependence, 81, 313-322.

Aharonovich, E., Nunes, E. y Hasin, D. (2003). Cognitive impairment, retention and abstinence among cocaine abusers in cognitivebehavioral treatment. Drug and Alcohol Dependence, 71, 207211.

Alderman, N., Dawson, K., Rutterford, N. A. y Reynolds P. J. (2001). A comparison of the validity of self-report measures amongst people with acquired brain injury: A preliminary study of the usefulness of Euro0ol-5D. Neuropsychological Rehabilitation, 11, 529-537.

Alexander, G. E., DeLong, M. R y Strick, P. L. (1986). Parallel organization of functionally segregated circuits linking basal ganglia and cortex. Annual Review of Neuroscience, 9, 357-381.

Amieva, H., Phillips, L. y Della Sala, S. (2003). Behavioral dysexecutive symptoms in normal aging. Brain and Cognition, 53, 129-132.

Barrash, J., Tranel, D. y Anderson, S. W. (2000). Acquired personality disturbances associated with bilateral damage to the ventromedial prefrontal region. Developmental Neuropsychology, 18, 355-381.

Bentler, P. M. (1990). Comparative fit indexes in structural models. Psychological Bulletin, 107, 238-246. 
Bentler, P. M. y Bonett, D. G. (1980). Significant tests and goodness of fit in the analysis of covariance structures. Psychological Bulletin, 88, 588-606.

Berg, E. A. (1948). A simple objective technique for measuring flexibility in thinking. Journal of General Psychology, 39, 15-22.

Bollen, K. A. (1989). Structural equations with latent variables. Nueva York: Wiley \& Sons.

Brislin, R. W. (1986). The wording and translation of research instruments. En W. Lonner y J. Berry (Eds.), Field methods in cross-cultural research (pp. 137-164). Beverly Hills, CA: Sage.

Browne, M. W. y Cudeck, R. (1993). Alternative ways of assessing model fit. En K.A. Bollen and J.S. Long (Eds). Testing structural equation models (pp 136-162). Newbury Park: Sage.

Burgess, P. W., Alderman, N., Evans, J., Emslie, H. y Wilson, B. (1998). The ecological validity of tests of executive function. Journal of the International Neuropsychological Society, 4, 547-558.

Carmines, E. y Mclver, J. (1981) Analyzing models with unobserved variables: analysis of covariance structures. En G. Bohrnstedt y E. Borgatta. (Eds), Social Measurement: Current Issues. Beverly Hills CA: Sage.

Carmines, E. G. y Zeller, R. A. (1979). Reliability and validity assessment. Londres: Sage.

Cattell, R. B. (1978). The scientific use of factor analysis in behavioural and life sciences. Nueva York: Plenum Press.

Chamberlain, E. (2003). Behavioural Assessment of the Dysexecutive Syndrome (BADS). Journal of Occupational Psychology, Employment and Disability, 5, 33-37.

Chan, R. C. K. (2001). Dysexecutive symptoms among a non-clinical sample: A study with the use of the Dysexecutive Questionnaire. British Journal of Psychology, 92, 551-565.

Chan, R. C. K., Hoosain, R. y Lee, T. M. C. (2002). Reliability and validity of the Cantonese version of the test of everyday attention among normal Hong Kong Chinese: a preliminary report. Clinical Rehabilitation, 16, 900-909.

Chaytor, N., Schmitter-Edgecombe, M. y Burr, R. (2006). Improving the ecological validity of executive functioning assessment. Archives of Clinical Neuropsychology, 21, 217-227.

Chaytor, N. y Schmitter-Edgecombe, M. (2007). Fractionation of the dysexecutive syndrome in a heterogeneous neurological sample: Comparing the Dysexecutive Questionnaire and the Brock Adaptive Functioning Questionnaire. Brain Injury, 21, 615-621.

Chico, E., Tous, J. M., Lorenzo, U. y Vigil, A. (2003). Spanish adaptation of Dickman's Impulsivity Inventory: its relationship to Eysenck's personality questionnaire. Personality and Individual Differences, 35, 1883-1892.

Cullen, B., Coen, R. F., Lynch, C. A, Cunningham, C. J., Coakley, D., Robertson, I. H et al. (2005). Repetitive behaviour in Alzheimer's disease: description, correlates and functions. International Journal of Geriatric Psychiatry, 20,686 - 693.

Cummings, J. L. (1993). Frontal subcortical circuits and human behavior. Archives of Neurology, 50, 873-880.

Cummings, J. L., Mega, M., Gray, K., Rosenberg-Thompson, S., Carusi, D. A. y Gornbein, J. (1994). The Neuropsychiatric Inventory: comprehensive assessment of psychopathology in dementia. Neurology, 44, 2308-2314.

Dickman, S. J. (1990). Functional and dysfunctional impulsivity: Personality and cognitive correlates. Journal of Personality and Social Psychology, 58, 95-102.
Eslinger, P. J. y Damasio, A. R. (1985). Severe disturbance of higher cognition after bilateral frontal lobe ablation: patient EVR. Neurology, 35, 1731-1741.

Fishbein, D. H., Krupitsky, E., Flannery, B. A., Langevin, D. J., Bobashev, G., Verbitskaya, E. et al. (2007). Neurocognitive characterizations of Russian heroin addicts without a significant history of other drug use. Drug and Alcohol Dependence, 90, 25-38.

Forcada, R., Pardo, N. y Bondia, B. (2006). Impulsividad en dependientes de cocaina que abandonan el consumo. Adicciones, 18, 111-118.

Giancola, P. R. y Mezzich, A. C. (2003). Executive functioning, temperament, and drug use involvement in adolescent females with a substance use disorder. Journal of Child Psychology \& Psychiatry \&t Allied Disciplines, 44, 857-866.

Gioia, G. A., Isquith, P. K., Guy, S. C. y Kenworthy, L. (2000). Behavior rating inventory of executive function. Neuropsychology, Development and Cognition, 6, 235-238.

Goldstein, R. Z., Leskovjan, A. C., Hoff, A. L., Hitzemann, R, Bashan, F., Khalsa, S. S., Wang, G. J., Fowler, J. S. y Volkow, N. D. (2004). Severity of neuropsychological impairment in cocaine and alcohol addiction: association with metabolism in the prefrontal cortex. Neuropsychologia, 42, 1447-1458.

Goodman, L. (1961). Snowball sampling. Annals of Mathematical Statistics, 32, 148.

Gorsuch, R. L. (1983). Factor analysis. Hillsdale, NJ: Lawrence Erlbaum. Grace, J. y Malloy, P. F. (2001). Frontal Systems Behavior Scale (FrSBe): professional manual. Lutz, FL: Psychological Assessment Resources.

Grace, J., Stout, J. C. y Malloy, P. F. (1999). Assessing frontal behavior syndromes with the Frontal Lobe Personality Scale. Assessment, 6, 269-284.

Hair, J. F., Anderson, R. E., Tatham, R. L. y Black, W. C. (1998). Multivariate Data Analysis ( $5^{\text {th }}$ Ed.). Upper Saddle River: Prentice-Hall.

Hart, T., Whyte, J., Kim, J. y Vaccaro, M. (2005). Executive function and self-awareness of real-world behavior and attention deficits following traumatic brain injury. Journal of Head Trauma Rehabilitation, 20, 333-347.

Hattie, J. (1984). Methodology review: Assessing unidimensionality of tests and items. Applied Psychological Measurement, 20, 1-14.

Hattie, J. (1985). An empirical study of the various indices for determining unidimensionality. Multivariate Behavioral Research, 19, 49-78.

Heffernan, T., Ling, J. y Bartholomew, J. (2004). Self-rated prospective memory and central executive deficits in excessive alcohol users. Irish Journal of Psychological Medicine, 21, 122-124.

Hester, R. y Garavan, H. (2004). Executive dysfunction in cocaine addiction: evidence for discordant frontal, cingulate, and cerebellar activity. Journal of Neuroscience, 24, 11017-11022.

Hinson, J. M., Jameson, T. L. y Whitney, P. (2003). Impulsive decision making and working memory. Journal of Experimental Psychology, 29, 298-306.

Hu, L. y Bentler, P. (1995). Evaluating model fit. En R. Hoyle (ed.), Structural equation modelling: concepts, issues and applications (pp. 76-99). Thousand Oaks, CA: Sage Publications.

James, L. R., Muliak, S. A. y Brett, J. (1982). Causal analysis: Models, assumptions and data. Beverly Hills, CA: Sage. 
Kertesz, A., Davidson, W. y Fox, H. (1997). Frontal behavioral inventory: diagnostic criteria for frontal lobe dementia. Canadian Journal of Neurological Sciences, 24, 29-36.

Kline, P. (1994). An easy guide to factor analysis. Londres: Routledge.

Landa, N., Fernández-Montalvo, J. y Tirapu, J. (2004). Alteraciones neuropsicológicas en el alcoholismo: una revisión sobre la afectación de la memoria y las funciones. Adicciones, 16, 41-52.

Landa, N., Fernández-Montalvo, J., Tirapu, J. López-Goñi, J., Castillo, A. y Lorea, I. (2006). Alteraciones neuropsicológicas en alcohólicos: un estudio exploratorio. Adicciones, 18, 49-60.

Larson, M. J., Perlstein, W. M., Demery, J. A. y Stigge-Kaufman, D. A. (2006). Cognitive control impairments in traumatic brain injury. Journal of Clinical and Experimental Neuropsychology, 28, 968-986.

Malloy, P. y Grace, J. A. (2005). Review of rating scales for measuring behavior change due to frontal systems damage. Cognitive and Behavioral Neurology, 18, 18-27.

Mathias, J. L. (2003). Neurobehavioral functioning of persons with Parkinson's disease. Applied Neuropsychology, 10, 57-68.

Mega, M. S. y Cummings, J. L. (1994). Frontal subcortical circuits and neuropsychiatric disorders. Journal of Neuropsychiatry and Clinical Neurosciences, 6, 358-370.

Mooney, B., Walmsley, C. y McFarland, K. (2006). Factor analysis of the Self-Report Dysexecutive (DEX-S) Questionnaire. Applied Neuropsychology, 13, 12-18.

Nunnally, J. C. (1978). Psychometric theory (2nd ed.). Nueva York: McGraw-Hill.

Pedhazur, E. J. (1997). Multiple regression in behavioral research: explanation and prediction. Fort Worth, TX: Harcourt Brace College Publishers

Pedrero, E. J., Ruiz Sánchez de León, J. M., Llanero, M., Rojo, G., Olivar, Á. y Puerta, C. (en prensa). Sintomatología frontal en adictos a sustancias en tratamiento mediante la versión española de la Escala de Comportamiento Frontal. Revista de Neurología, en prensa.

Prosser, J., Cohen, L. J., Steinfeld, M., Eisenberg, D., London, E. D. y Galynker, I. I. (2006). Neuropsychological functioning in opiate-dependent subjects receiving and following methadone maintenance treatment. Drug and Alcohol Dependence, 84, 240-247.

Reay, J. L., Hamilton, C., Kennedy, D. O. y Scholey, A. B. (2006). MDMA polydrug users show process-specific central executive impairments coupled with impaired social and emotional judgement processes. Journal of Psychopharmacology, 20, 385388.

Reitan, R. M. (1958). Validity of the Trail Making Test as an indicator of organic brain damage. Perceptual and Motor Skills, 8, 271-286.

Schilt, T., de Win, M. M. L., Koeter, M., Jager, G., Korf, D. J., van den Brink, W. et al. (2007). Cognition in novice ecstasy users with minimal exposure to other drugs: a prospective cohort study. Archives of General Psychiatry, 64, 728-736.

Selby, M. J. y Azrin, R. L. (1998). Neuropsychological functioning in drug abusers. Drug and Alcohol Dependence, 50, 39-45.

Shallice, T. y Burgess, P. W. (1991) Higher-order cognitive impairments and frontal lobe lesions in man. In: H.S. Levin, H.M. Eisenberg y A.L. Benton (Eds.), Frontal Lobe Function and Dysfunction (pp. 125-138). Nueva York: Oxford University Press.

Stroop, J. R. (1935). Studies of interference in serial verbal reactions. Journal of Experimental Psychology, 18, 643-662
Stuss, K. H. y Benson, D. F. (1984). Neuropsychological studies of the frontal lobes. Psychological Bulletin, 95, 3-28.

Teichner, G., Horner, M. D., Roitzsch, J. C., Herron, J. y Thevos, A. (2002). Substance abuse treatment outcomes for cognitively impaired and intact outpatients. Addictive Behaviors, 27, 751-763.

Verdejo, A., Lawrence, A. J. y Clark, L. (2008). Impulsivity as a vulnerability marker for substance use disorders: Review of findings from high-risk research, problem gamblers and genetic association studies. Neuroscience \&t Biobehavioral Reviews, 32, 777-810.

Verdejo, A., López-Torrecillas, F., Orozco, C. y Pérez, M. (2002). Impacto de los deterioros neuropsicológicos asociados al consumo de sustancias sobre la práctica clínica con drogodependientes. Adicciones, 14, 345-370.

Verdejo, A. y Pérez-García, M. (2007). Ecological assessment of executive functions in substance dependent individuals. Drug and Alcohol Dependence, 90, 48-55.

Walley, R. M. y Donaldson, M. D. (2005). An investigation of executive function abilities in adults with Prader-Willi syndrome. Journal of Intellectual Disability Research, 49, 613-625.

Wang, Y., Chan, R. C. K. y Deng, Y. Y. (2006). Examination of postconcussion-like symptoms in healthy college students: relationships to subjective and objective neuropsychological function performance. Archives of Clinical Neuropsychology, 21, 339-347.

Wheaton, B., Muthén, B., Alwin, D. F. y Summers, G. F. (1977). Assessing reliability and stability in panel models. EnHeise, D.R. (Ed.) Sociological methodology 1977. San Francisco: JosseyBass, 84-136.

Whitney, P., Hinson, J. M. y Jameson, T. L. (2006). From executive control to self-control: predicting problem drinking among college students. Applied Cognitive Psychology, 20, 823-835.

Wilson, B. A., Alderman, N., Burgess, P. W., Emslie, H. y Evans, J. J. (1996). Behavioural assessment of the Dysexecutive Syndrome. Bury St. Edmunds, UK : Thames Valley Test Company.

Wood, R. LI. y Liossi, C. (2006). The ecological validity of executive tests in a severely brain injured sample. Archives of Clinical Neuropsychology, 21, 429-437. 


\section{Apéndice 1. İtems de la versión española del Dysexecutive Questionnaire (DEX-Sp)}

Tengo problemas para entender lo que otros quieren decir, aunque digan las cosas claramente.

2 Actúo sin pensar, haciendo lo primero que me pasa por la cabeza.

3 A veces hablo sobre cosas que no han ocurrido en realidad, aunque yo creo que si han pasado.

4 Tengo dificultad para pensar cosas con antelación o para planificar el futuro.

A veces me pongo demasiado excitado con ciertas cosas y en esos momentos me paso un poco de la raya.

6 Mezclo algunos episodios con otros, y me confundo al intentar ponerlos por orden.

Tengo dificultades para ser consciente de la magnitud de mis problemas y soy poco realista respecto a mi futuro.

8 Estoy como aletargado, o no me entusiasmo con las cosas.

9 Hago o digo cosas vergonzosas cuando estoy con otras personas.

10 Tengo muchas ganas de hacer ciertas cosas en un momento dado, pero al momento ni me preocupo de ellas.

11 Tengo dificultad para mostrar mis emociones.

12 Me enfado mucho por cosas insignificantes.

13 No me preocupo sobre cómo tengo que comportarme en ciertas situaciones.

Me resulta dificil dejar de decir o hacer repetidamente ciertas cosas, una vez que he empezado a hacerlas.

15 Tiendo a ser bastante activo, y no puedo quedarme quieto por mucho tiempo.

Me resulta dificil cortarme de hacer algo incluso aunque sepa que no deberia hacerlo.

17 Digo una cosa pero después no actúo en consecuencia, no la cumplo.

18 Me resulta dificil centrarme en algo, y me distraigo con facilidad.

19 Tengo dificultades para tomar decisiones, o decidir lo que quiero hacer.

20 No me entero, o no me interesa, lo que opinen otros sobre mi comportamiento. 